\title{
Full-Information Item Factor Analysis: Applications of EAP Scores
}

\author{
Eiji Muraki \\ National Opinion Research Center \\ George Engelhard, Jr. \\ Emory University
}

The full-information item factor analysis model proposed by Bock and Aitkin (1981) is described, and some of the characteristics of expected a posteriori (EAP) scores are illustrated. Three simulation studies were conducted to illustrate the model, and an application of full-information item factor analysis to a set of real data is described.

Factor analysis has been a powerful and indispensible tool for exploring underlying relationships among a set of continuous variables. The procedure generally begins with the analysis of the productmoment correlations among variables under some restrictions. In the case of dichotomous variables, the matrix of tetrachoric correlation coefficients is commonly used in place of the product-moment correlation coefficients. Divgi (1979) developed a fast and generally accurate computational algorithm. However, the coefficients become unstable inevitably as they approach extreme values. The coefficient cannot be computed from a four-fold table with a zero frequency in any cell, and an appropriate value must be imputed. These situations frequently occur in the analysis of cognitive ability and achievement tests, especially when the difficulties of the items in a test vary to a great extent. In addition to the computational difficulties of tetrachoric correlation coefficients, the sample matrix of tetrachoric correlation coefficients is seldom positive definite so that some smoothing process must be performed on the original matrix before common factor analysis is applied.

Solutions to the problems encountered with tetrachoric factor analysis have been provided by Christoffersson (1975) and Muthén (1978). However, their approach to item factor analysis becomes computationally heavier as the number of items increases (Muthén, 1984). Therefore, their method is of limited practical use for analyzing achievement and cognitive test responses since these tests generally consist of a sizable number of items.

Bock and Aitkin (1981) developed dichotomous factor analysis based on multidimensional item response models. Their method starts with the distinct item response vectors of the examinees. Since the procedure uses all the information available in the matrix of dichotomously scored response patterns, it is called "full-information" item factor analysis (Bartholomew, 1980). Unlike other approaches to te-

APPLIED PSYCHOLOGICAL MEASUREMENT

Vol. 9, No. 4, December 1985, pp. 417-430

(C) Copyright 1985 Applied Psychological Measurement Inc.

0146-6216/85/040417-14\$1.95 
trachoric item factor analysis, Bock and Aitkin's method is not limited by the number of items and is applicable to exploring the dimensionality of long tests.

Bock and Aitkin (1981) also discussed three methods of estimating factor scores: the maximum likelihood (ML) estimator, the maximum a posteriori (MAP) estimator, and the expected a posteriori (EAP) estimator. Bock and Mislevy (1982) have shown that the EAP scores have unusually good properties, which are not found with the other estimators.

Bock, Gibbons, and Muraki (1985) have applied full-information item factor analysis to the data analysis of the Armed Services Vocational Aptitude Battery (ASVAB). In their data analyses, changes in the chi-square fit statistics successfully indicated the interpretable dimensionality of each subtest of the ASVAB, and the content of test items reflected the extracted factors.

The main purpose of this study was to apply the full-information item factor analysis method to item response data which are expected to be multidimensional. In order to show the potential advantages of full-information item factor analysis, three simulation studies were conducted.

In the first simulation study, a comparison was made between the results of full-information item factor analysis and a conventional tetrachoric factor analysis. Several desirable properties of the EAP scores for the purpose of data analysis were shown in the second and third simulation studies. Finally, full-information item factor analysis was used to analyze four of the Affective Outcomes of Schooling (AOS) Scales developed by Engelhard (1985). The analysis of this data set illustrates a typical application of this method and EAP scores in educational and psychological research.

\section{The Model}

\section{Description of the Item Factor Analysis Model}

In adapting Thurstone's multiple factor model to dichotomous data, Bock and Aitkin (1981) considered an unobservable response process, $y_{i j}$, for person $i$ and item $j$, to be a linear combination of $m$ normally distributed latent variables, $\boldsymbol{\theta}$, weighted by the factor loadings, $\boldsymbol{\alpha}$; that is,

$y_{i j}=\alpha_{j 1} \theta_{1 i}+\alpha_{j 2} \theta_{2 i}+\ldots .+\alpha_{j m} \theta_{m i}+\delta_{i}$.

For a randomly sampled person, it is assumed that $\boldsymbol{\theta}, \mathbf{y}$, and $\boldsymbol{\delta}$ are multivariate normal:

$\boldsymbol{\theta} \sim N(\mathbf{O}, I)$,

$\mathbf{y} \sim N(\mathbf{O}, I)$,

and

$\boldsymbol{\delta} \sim N\left(\mathbf{O}, \mathbf{D}_{\mathbf{s}}\right)$.

Assumptions 2 and 3 imply that the diagonal elements of $\mathbf{D}_{\sigma}$ in Assumption 4 , denoted as $\sigma_{j}^{2}$, are

$\sigma_{j}^{2}=1-\sum_{k=1}^{m} \alpha_{j k}^{2}$

The classical factor analysis model for continuous variables assumes that the response process is directly observable. In contrast to this model, the factor analysis model for categorical variables assumes that the unobservable response process $y_{i j}$ is latent and realized only as a vector of dichotomous response variables, $\mathbf{x}_{i}=\left(x_{i 1}, x_{i 2}, \ldots, x_{i n}\right)$, according to the following item response mechanism:

$x_{i j}=\left\{\begin{array}{l}1 \text { if } y_{i j} \geqslant \gamma_{j} \\ 0 \text { if } y_{i j}<\gamma_{j}\end{array}\right.$,

where $\gamma_{j}$ is a threshold parameter associated with item $j$. Therefore, the probability of a positive response by person $i$ to item $j$, given the individual's $m$-dimensional latent ability, $\boldsymbol{\theta}_{i}$, is 
$P\left(x_{i j}=1 \mid \boldsymbol{\theta}_{i}\right)=\frac{1}{\sqrt{(2 \Pi)} \sigma} \int_{\gamma_{j}}^{\infty} \exp \left[-\frac{1}{2}\left(\frac{y_{i j}-\sum \alpha_{j k} \theta_{k i}}{\sigma_{j}}\right)^{2}\right] d y_{i j}$.

Introducing the change in the variable

$t=\left(y_{i j}-\sum \alpha_{j k} \theta_{k i}\right) / \sigma_{j}$,

the result is

$d y_{i j}=\sigma_{j} d t$,

and, when

$y_{i j}=\gamma_{j}$

and

$t=\left(\gamma_{j}-\sum \alpha_{j k} \theta_{k i}\right) / \sigma_{j}$,

the model of Equation 7 can then be rewritten with slope parameters $a_{j k}$ and location parameters $c_{k}$ as follows:

$$
\begin{aligned}
P\left(x_{i j}=1 \mid \boldsymbol{\theta}_{i}\right) & =\int_{-\left(\Sigma a_{j k} \theta_{k i}+c_{j}\right)}^{\infty} \phi(t) d t \\
& =\Phi_{j}\left(\boldsymbol{\theta}_{i}\right),
\end{aligned}
$$

where

$a_{j k}=\alpha_{j k} / \sigma_{j}$,

and

$c_{j}=-\gamma_{j} / \sigma_{j}$.

From Equations 5, 12, and 13, the following formulas are obtained to convert the slope and location estimates to factor loadings and threshold values:

$\alpha_{j k}=a_{j k} / d_{j}$,

and

$\gamma_{j}=-c_{j} / d_{j}$,

where

$d_{j}^{2}=\left(1+\sum a_{j k}^{2}\right)$.

A guessing or lower asymptote parameter, $g_{j}$, can also be incorporated into the model of Equation 11 as follows:

$\Phi_{j}^{*}(\boldsymbol{\theta})=g_{j}+\left(1-g_{j}\right) \Phi_{j}(\boldsymbol{\theta})$.

The iterative procedure developed for obtaining the parameter estimates in the multidimensional item response model (Equation 11) is based on marginal maximum likelihood estimation and the EM algorithm of Dempster, Laird, and Rubin (1977). This method is implemented in the TESTFACT program of Wilson, Wood, and Gibbons (1984). At each step of the analysis, the solution from a principal factor analysis for the current number of factors provides initial estimates of the parameters. After the final estimation cycle is completed, estimated values are listed in the form of factor loadings and thresholds, as well as a set of slope and intercept parameter estimates.

The TESTFACT program provides the option for conducting a stepwise analysis. When the number of possible response patterns is larger than the sample size and the table of realized frequencies is sparse, the likelihood ratio fit statistics are inaccurate and unreliable. Haberman (1977) shows, however, that the difference in these statistics for alternative models is distributed in large samples as chi-square. The chisquare fit statistics for each solution and the reduction from the previous solution in the stepwise analysis, therefore, provide a large sample test of significance of factors added to the model. 
The factor loadings from the full-information solution are rotated orthogonally to the Varimax criterion and then obliquely to the Promax criterion. Because the computational time of the full-information factor solution increases exponentially in the number of factors, the present version of the TESTFACT program is limited to a maximum of five factors.

\section{The EAP Estimator}

The EAP estimator of the factor scores for the $k$ th dimension is the mean of the posterior distribution of $\boldsymbol{\theta}$ for that dimension, which is expressed by:

$$
\begin{aligned}
\tilde{\theta}_{i k} & =E\left(\theta_{i k} \mid \mathbf{x}_{i}\right) \\
& =\int_{\boldsymbol{\theta}} \theta_{k} P\left(\boldsymbol{\theta} \mid \mathbf{x}_{i}\right) d \boldsymbol{\theta} \\
& =\int_{\boldsymbol{\theta}} \frac{\theta_{k} f\left(\mathbf{x}_{i} \mid \boldsymbol{\theta}\right) g(\boldsymbol{\theta})}{h\left(\mathbf{x}_{i}\right)} d \boldsymbol{\theta},
\end{aligned}
$$

where

$f\left(\mathbf{x}_{i} \mid \boldsymbol{\theta}\right)=\prod_{j=1}^{n} \Phi_{j}(\boldsymbol{\theta})^{x_{i j}}\left[1-\Phi_{j}(\boldsymbol{\theta})\right]^{1-x_{i j}}$,

and

$h\left(\mathbf{x}_{i}\right)=\int_{\boldsymbol{\theta}} f\left(\mathbf{x}_{i} \mid \boldsymbol{\theta}\right) g(\boldsymbol{\theta}) d \boldsymbol{\theta}$.

In the Bayesian context, $f(\cdot)$ is called the "likelihood function," and $g(\cdot)$ the "prior" probability density. The $h(\cdot)$ is the marginal probability of the response vector $\mathbf{x}_{i}$, which normalizes the posterior probability. The posterior probability $p\left(\boldsymbol{\theta} \mid \mathbf{x}_{i}\right)$ is the conditional density of $\boldsymbol{\theta}$ given the $i$ th response vector $\mathbf{x}_{i}$. Therefore, the posterior density contains all the information available about $\boldsymbol{\theta}_{i}$ that is expressed by the distinct response pattern $\mathbf{x}_{i}$.

The EAP estimator is known to be biased when the number of items is finite. However, unlike the maximum likelihood estimator (MLE), the EAP scores can be computed for response patterns with all correct and all incorrect responses.

In the TESTFACT program, Equation 18 is evaluated numerically by $m$-fold Gauss-Hermite product quadrature using the nodes and weights for one-dimensional quadrature (Stroud \& Sechrest, 1966). The computation of the EAP scores does not require any iterative procedure. If the parameter values in the item response model $\Phi(\theta)$ are known and the proper quadrature nodes and weights are evaluated beforehand, the EAP scores based on any subset of items in a test can be computed straightforwardly. This advantage is pointed out by Bock and Mislevy (1982) for the application of computerized adaptive testing, in which additional information about the examinee's ability needs to be accommodated continuously during a series of item presentations. In the context of a data analytic procedure, this desirable property allows researchers to compare the EAP scores of examinees based on different sets of sampled items (Bock, Mislevy, \& Woodson, 1982).

\section{Simulation Studies}

\section{Comparison Between Tetrachoric and Full-Information Factor Analyses}

Simulated data sets. In the first simulation study, full-information item factor analysis was compared with a conventional tetrachoric factor analysis. First, a set of 2,000 pairs of independently and identically 
distributed normal variates was generated. Schrage's (1979) method and the composite method of Ahrens and Dieter (1972) were used to generate uniform random variables and transform them to unit normal deviates, respectively. Second, by using those normal random variates as latent variables, $\boldsymbol{\theta}$, the response vectors for 22 items were generated according to two types of two-dimensional normal ogive item response models. One was the model without a guessing parameter, and the other was the model with a guessing parameter. A normally distributed error term was assumed for both models.

For the first 11 items, the first factor loadings, $\alpha_{j 1}$, were uniformly set as .707 and the second factor loadings, $\alpha_{j 2}$, as 0.0 . For the second 11 items, the $\alpha_{j 1}$ s were uniformly set as 0.0 and $\alpha_{j 2}$ s as .707 . For both sets of items, the threshold values, $\gamma_{j}$, were equally spaced from -2.0 to +2.0 . The lower asymptotes, $g_{j}$, for the guessing model were uniformly set as .2 .

After generating two sets of 2,000 response vectors, a sample of 1,000 response vectors was selected from each of the original data sets. Thus, the following four simulated data sets were produced:

1. 2,000 response vectors without guessing effects,

2. 1,000 response vectors without guessing effects,

3. 2,000 response vectors with guessing effects, and

4. 1,000 response vectors with guessing effects.

The entire process of data generation described above was repeated three times by supplying other seeds to a random number generator. Consequently, four types of 12 data sets were prepared for the subsequent analyses.

Tetrachoric and full-information factor analyses. Tetrachoric factor analyses of the 12 data sets were performed. Since the range of threshold values was set quite apart, the success and failure rates for items at the extreme ends of the difficulty scale became extremely high. Consequently, about $10 \%$ of the elements in each of the tetrachoric matrices had to be imputed according to the Centroid method (Bock et al., 1985). After further correcting the observed matrices of tetrachoric correlations to be semipositive definite, the MINRES method (Harman, 1976) was performed to obtain principal factor loadings. These tetrachoric factor solutions were used as the starting values to the iteration cycles of the full-information factor analysis.

For the tetrachoric factor analyses of response data sets with guessing effects, Carroll's (1945) correction method was applied to the proportions in the four-fold tables. For the full-information analysis, the guessing model of Equation 17 was used. In both analyses, lower asymptotes were supplied as known constants, .2, and not estimated.

The solutions from both tetrachoric and full-information methods were rotated orthogonally according to the Varimax criterion (Kaiser, 1958). The original factor loadings, .707 and 0.0 , were subtracted from the recovered Varimax factor loadings.

Results and discussion. Means and standard deviations of the differences are plotted along the original threshold values, $\gamma_{j}$, in Figures 1 and 2 . Among 11 threshold levels, only six levels are presented for the sake of clarity. The number of observations at each threshold level is 12 .

The full-information method generally does better in recovering original factor loadings in both noguessing and guessing data sets. Their means of differences are closer to 0 (61\% of comparisons) and their standard deviations are almost always smaller (91\% of comparisons) compared to those in the tetrachoric factor solutions. Especially at the extreme threshold levels, the full-information factor method clearly outperforms the tetrachoric factor method. The tetrachoric factor analysis tends to produce very low estimates for extremely easy $\left(\gamma_{j}=-.2\right)$ and extremely difficult items $\left(\gamma_{j}=.2\right)$. However, when the sample size is large enough and the probabilities of dichotomous item responses are not so extreme, the tetrachoric method can produce reasonably good estimates. As shown in Figures 1 and 2, both the tetrachoric and the full-information factor solutions for moderately difficult items are virtually indistinguishable. 
Figure 1

Means and Standard Deviations of Differences Between Estimated and Original Factor Loadings for Data without Guessing Effects

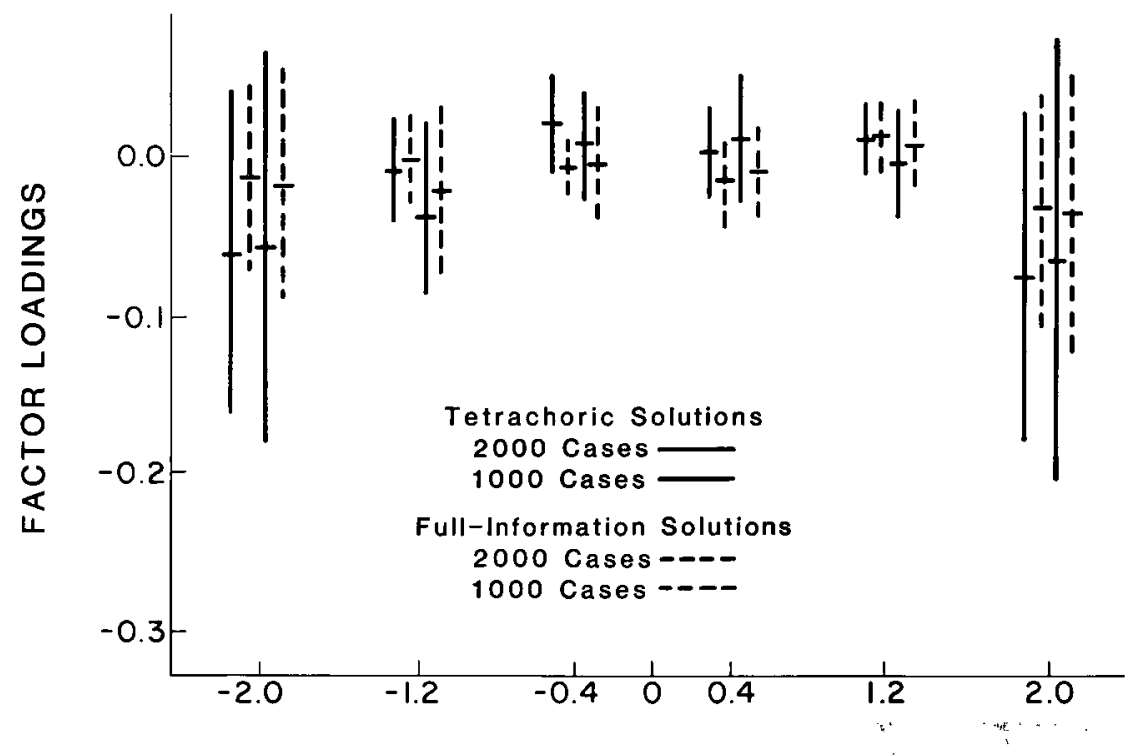

THRESHOLD LEVEL

Although both Carroll's (1945) correction method and the model with a guessing parameter seem to work well for removing the effect of chance success from item parameter estimation, both methods tend to produce less stable estimates. The simultaneous estimation of guessing parameters is not implemented in the current version of the TESTFACT program, but it is planned to be included in a future version of the program. An alternative model to the current guessing model is also being considered. Fullinformation factor analysis is capable of treating guessing effects more flexibly than the tetrachoric factor analysis. Further studies, including a new parameterization of the guessing model, are necessary to discuss advantages of the full-information factor analysis for the treatment of guessing effects. Unlike the tetrachoric factor method, the full-information factor method uses maximum likelihood (ML) estimation, and its estimator is known to be efficient. The present simulation study failed to demonstrate this desirable characteristic of ML estimators in comparison to the tetrachoric solutions. Both methods equally improve their estimates at the moderate levels of difficulty as the number of observations increases.

The full-information method can maximally use information available in distinct response patterns to compute item parameter estimates. The method can provide more accurate estimates than tetrachoric factor analysis, especially when item difficulties vary to a great extent. However, this method involves heavy iterative computations. For example, while the TESTFACT program needed 2 minutes of CPU time (IBM 3081 computer) for the complete tetrachoric factor analysis of 12 data sets, it required 81 minutes for the ML parameter estimates to converge at the accuracy level of .001 . In addition to the development of better acceleration methods to shorten the iterative process, the method of supplying more accurate starting parameter values must be developed, especially when guessing effects and missing responses are present.

Bock et al. (1985) have compared the solutions of the full-information item factor analysis with Muthén's (1978) generalized least square (GLS) solution based on the data from Quality of Life (Campbell, 
Converse, \& Rodgers, 1976). Although these two methods are computationally different, parameters in both solutions were quite similar and the differences of the chi-square fit statistics in the stepwise analysis were virtually identical. In this article, the comparison was made between a conventional tetrachoric factor analysis and full-information factor analysis based on the simple structure of simulated data sets. Further comparison studies about these methods incorporating a higher dimensional data structure, varied factor loadings, guessing effects, and a missing response treatment are necessary to reach final conclusions on the advantages and disadvantages of each method.

\section{Uncorrelated EAP Scores}

For the second simulation study, 2,000 pairs of independently and identically distributed random variables, $\boldsymbol{\theta} \sim \mathrm{N}(\mathbf{O}, I)$ were generated. The two-dimensional item parameter estimates of the Auto and Shop Information subtest in the ASVAB obtained by Mislevy and Bock (1984) were used to generate the simulated dichotomous item responses to 25 items. Assuming the guessing parameter to be constant, the item statistics for the normal ogive item response model (Equation 17) were calibrated. The two-dimensional Varimax factor loadings were computed from these item parameter estimates. The EAP scores in the twodimensional continuum, which are denoted $\tilde{\theta}_{1}$ and $\tilde{\theta}_{2}$, were computed from the item parameter estimates after the Varimax rotation. Twenty Gauss-Hermite quadrature points and weights in each dimension were used for the numerical integration.

Multivariate multiple regression analysis of the generated random variables $\boldsymbol{\theta}$ on the EAP estimates $\overline{\boldsymbol{\theta}}$ was performed. No significant quadratic or cubic term was detected. The regression function is

Figure 2

Means and Standard Deviations of Differences Between Estimated and Original Factor Loadings for Data with Guessing Effects

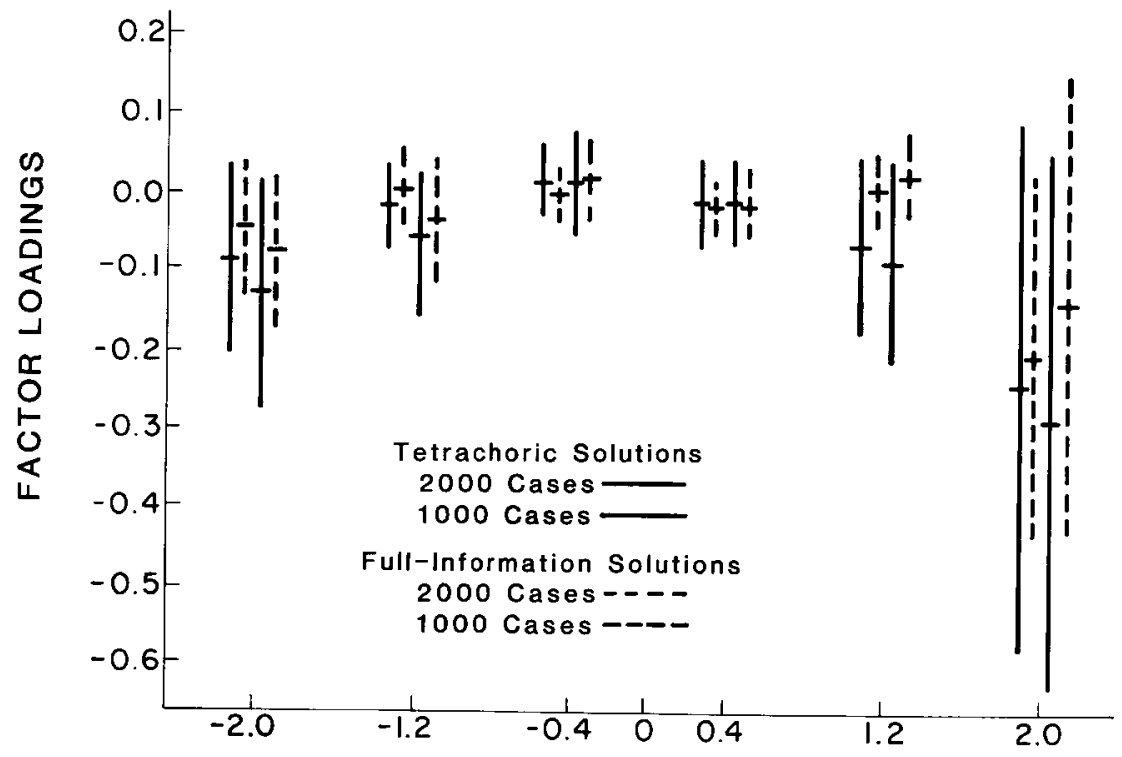

THRESHOLD LEVEL 
$\left[\begin{array}{l}\theta_{1} \\ \theta_{2}\end{array}\right]=\left[\begin{array}{l}.012 \\ .006\end{array}\right]+\left[\begin{array}{rr}.954 & -.029 \\ .036 & 1.019\end{array}\right]\left[\begin{array}{l}\tilde{\theta}_{1} \\ \tilde{\theta}_{2}\end{array}\right]+\left[\begin{array}{l}\varepsilon_{1} \\ \varepsilon_{2}\end{array}\right]$.

The actual values of the EAP scores depend on the particular choice of rotation. Nevertheless, the major and minor diagonals of the regression coefficient matrix become close to 1 and 0 , respectively. In other words, the EAP scores based on the Varimax solution are essentially uncorrelated. This is not generally true of other methods of computing factor scores (Harman, 1976).

The correlation between the simulated and estimated abilities was .672 and .778 for the first and second dimensions, respectively. When the same simulation procedure described above was repeated with 500 cases, the correlation dropped to .644 and .752 . This may be largely due to the poor convergence of item parameter estimation when the sample sizes are small. Small sample sizes become problematic in computing the EAP scores only when the item parameters must be estimated from the same observed responses. Otherwise, increasing the length of the test leads to more reliable EAP scores.

\section{Comparison Between ML and EAP Estimates}

For the third simulation study, a set of 30 unidimensional item estimates from the ASVAB Arithmetic subtest (Mislevy \& Bock, 1984) was split into 15 odd-numbered items and 15 even-numbered items. The 1,000 dichotomous item response vectors for each set of 30 items were generated based on the guessing model (Equation 17) and the same underlying unidimensional ability distribution. This experiment closely simulated the situation in which the same group of students takes two parallel forms of a test within a certain time interval. The ML estimates and the EAP estimates were computed from these sets of evennumbered and odd-numbered items, and they are plotted in Figures 3 and 4, respectively. Some plots that are approximately located on the same positions are hidden in the figures.

Since guessing effects were included in the item response model, a ceiling effect appears in both plots. In addition to ceiling effects, the plot of the ML estimates shows that the ability scores are more

Figure 3

MLE Estimates from Even- and Odd-Numbered Items

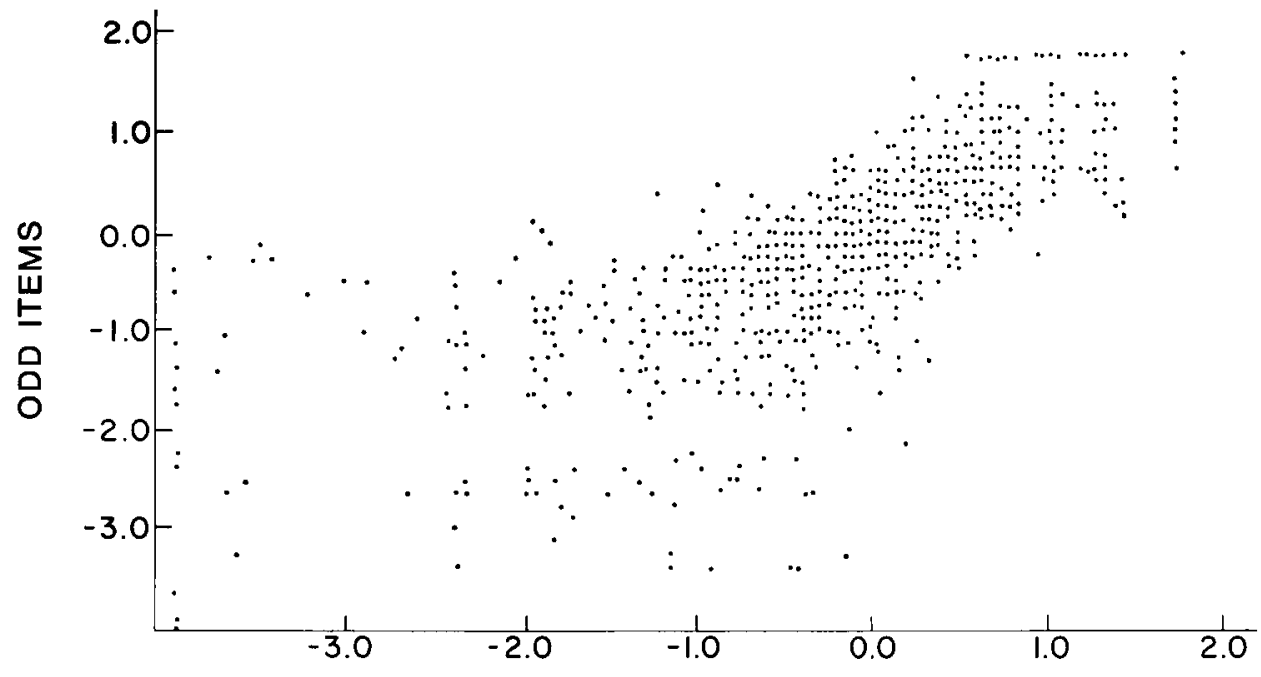

EVEN ITEMS 


\section{Figure 4}

EAP Estimates from Even- and Odd-Numbered Items

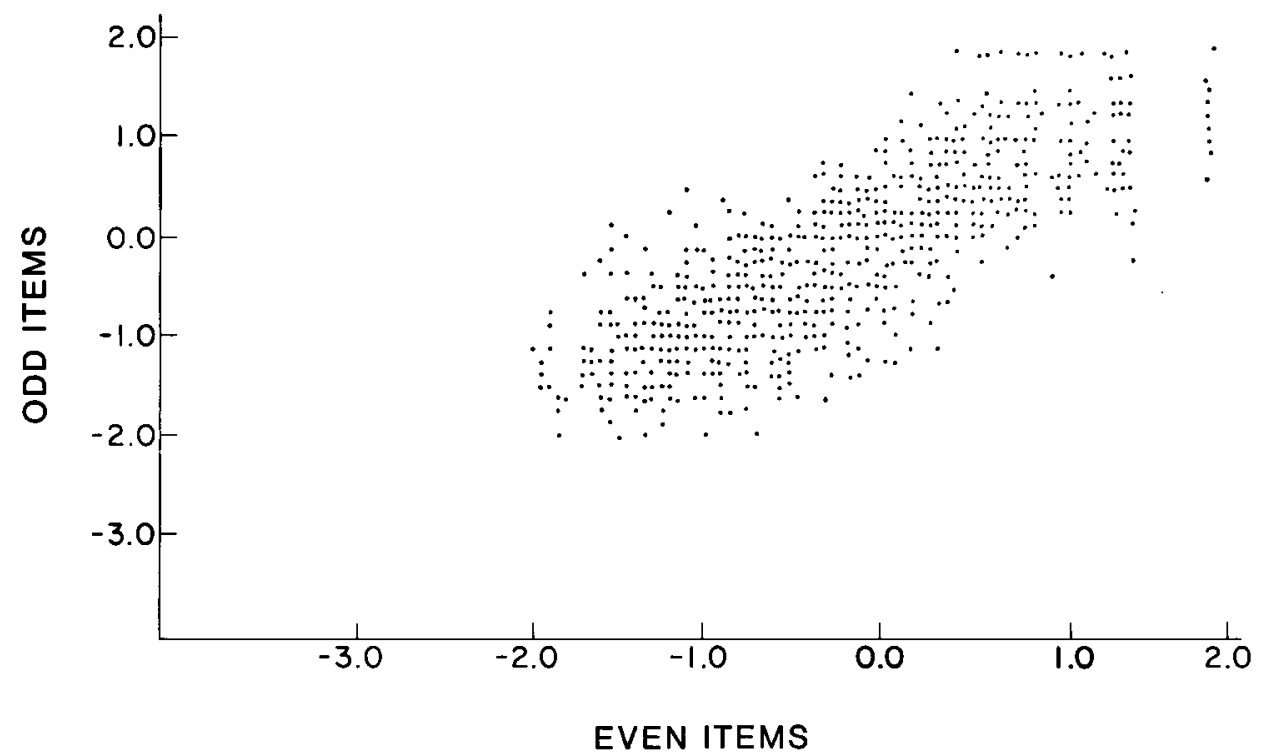

dispersed at the lower end of the continuum. This is because accurate ML estimates cannot be obtained for extreme scores. The unreliability of the ML estimator of the scores at the lower and higher ends of the ability scale may be accentuated when the test is not very long or highly heterogeneous guessing effects are found. The comparison between the ML estimates and the EAP estimates reveals, therefore, that the EAP estimator provides a more reliable reproduction of the simulated abilities than the ML estimator. This aspect of the EAP scores is important, since parallel forms are frequently required in educational and psychological testing.

\section{Applications}

\section{Measurement of Affective Outcomes of Schooling (AOS)}

In this section, 40 items were selected from the AOS Scales developed by Engelhard (1985). Items were selected from the Punctuality, Honesty, Cooperation, and Curiosity Scales. The items in the AOS Scales are scored dichotomously (yes/no), and there were 700 elementary school students in the data set.

\section{Factor Patterns of AOS Data}

A stepwise full-information item factor analysis was performed specifying a four-factor solution for the AOS data. The differences in the chi-square fit statistics of each solution are presented in Table 1. Although the reduction of the chi-square fit statistics seems to suggest that a model with more than four dimensions may provide a better fit to the data, a careful examination of the fourth factor loadings revealed that these loadings were minor and no interpretable configuration was found. Therefore, the three-factor solution was chosen as an appropriate model to describe the behavior of the AOS data. The threedimensional Varimax factor loadings are presented in Table 2. 
Table 1

Reduction in Chi-Square Fit Statistics

\begin{tabular}{lcc}
\hline $\begin{array}{c}\text { Additonal } \\
\text { Factors }\end{array}$ & $\begin{array}{c}\text { Reduction } \\
\text { in } \\
\text { Chi-Square }\end{array}$ & $\begin{array}{c}\text { Degrees } \\
\text { of } \\
\text { Freedom }\end{array}$ \\
\hline Second factor & 349.04 & 39 \\
Third factor & 259.25 & 38 \\
Fourth factor & 171.35 & 37 \\
\hline
\end{tabular}

The factor patterns in Table 2 indicate that the first factor loadings were generally high on most of the Honesty and Cooperation items. Some of the items with high loadings were

I understand that being honest is important.

I believe that working with other students is more important than competing with them.

I can tell when it is better to work together with other students than to compete with them.

The items with high loadings on the second factor came primarily from the Curiosity Scale. Some of the items with high loadings on this factor were

I try to find out all I can about the subjects that the teacher talks about in class.

Being curious about my classwork is important to me.

I enjoy looking for and trying out new ideas and projects.

Finally, some sample items with high loadings on the third factor were

I finish my classroom assignments when they are due.

I turn in my homework on time.

I finish my classwork on time, even when the teacher is not around.

The items with high loadings on this factor came primarily from the Punctuality Scale.

\section{Advantages of EAP Scores}

Full-information item factor analysis provides an objective basis for clarifying the distinction between what the item writer intends to measure and what the items actually appear to measure. At the stage of test or scale construction, full-information factor analysis provides a powerful method for examining the construct validity of each item. For example, one of the items in the Cooperation Scale ("I do not compete with other students for good grades.") had low factor loadings on all three dimensions. The item was worded negatively and this may have confused some of the examinees. This is an example of how fullinformation item factor analysis is quite sensitive to ill-behaved items.

Despite a small lack of accordance on a few items, the agreement between a prior classification of items into four scales and the results of the full-information item factor analysis is quite high. Items from the Honesty and Cooperation Scales define the first factor, items from the Curiosity Scale define the second factor, and items from the Punctuality Scale define the third factor. 
Table 2

Varimax Factor Loadings for the Three Factor Solution

\begin{tabular}{|c|c|c|c|c|}
\hline \multirow{2}{*}{$\begin{array}{l}\text { Scale } \\
\text { and } \\
\text { Item }\end{array}$} & \multirow[b]{2}{*}{ Threshold } & \multicolumn{3}{|c|}{ Factor } \\
\hline & & 1 & 2 & 3 \\
\hline \multicolumn{5}{|l|}{ Honesty } \\
\hline 1 & -1.564 & .595 & -.011 & .078 \\
\hline 2 & -1.643 & .500 & .253 & .160 \\
\hline 3 & -1.361 & .329 & .262 & .195 \\
\hline 4 & -.230 & .278 & -.028 & .118 \\
\hline 5 & -1.001 & .244 & .229 & .407 \\
\hline 6 & -.967 & .131 & .241 & .502 \\
\hline 7 & -1.432 & .412 & .371 & .343 \\
\hline 8 & -.566 & .253 & .317 & .465 \\
\hline 9 & -1.164 & .283 & -.051 & .097 \\
\hline 10 & -.860 & .512 & .066 & .269 \\
\hline \multicolumn{5}{|c|}{ Cooperation } \\
\hline 11 & -.757 & .454 & .024 & .020 \\
\hline 12 & -1.113 & .348 & .252 & .134 \\
\hline 13 & .238 & .095 & -.212 & .169 \\
\hline 14 & -.848 & .303 & .152 & .254 \\
\hline 15 & .197 & .208 & -.006 & -.084 \\
\hline 16 & -1.113 & .431 & -.009 & .152 \\
\hline 17 & -.801 & .541 & .032 & .116 \\
\hline 18 & -.988 & .249 & .356 & .060 \\
\hline 19 & -.660 & .267 & .164 & -.109 \\
\hline 20 & -1.088 & .487 & .219 & .279 \\
\hline \multicolumn{5}{|l|}{ Curiosity } \\
\hline 21 & -1.815 & .219 & .321 & .357 \\
\hline 22 & -.591 & .146 & .340 & .172 \\
\hline 23 & -.519 & .069 & .258 & .094 \\
\hline 24 & -.945 & -.021 & .494 & .052 \\
\hline 25 & -.633 & -.101 & .703 & .100 \\
\hline 26 & -.181 & -.116 & .612 & -.018 \\
\hline 27 & -.846 & .136 & .541 & .125 \\
\hline 28 & -.954 & .163 & .580 & .097 \\
\hline 29 & .210 & -.087 & .437 & .017 \\
\hline 30 & -1.225 & .157 & .519 & .217 \\
\hline \multicolumn{5}{|c|}{ Punctuality } \\
\hline 31 & -2.062 & .262 & .225 & .292 \\
\hline 32 & -1.267 & .271 & .497 & .252 \\
\hline 33 & -1.473 & .425 & .220 & .231 \\
\hline 34 & -.525 & .320 & .158 & .385 \\
\hline 35 & -1.791 & .272 & .372 & .203 \\
\hline 36 & -.824 & .196 & .401 & .431 \\
\hline 37 & -.731 & .015 & .015 & .838 \\
\hline 38 & -.906 & .208 & .105 & .802 \\
\hline 39 & -1.065 & .256 & .509 & .399 \\
\hline 40 & -.448 & -.002 & .061 & .855 \\
\hline
\end{tabular}


After obtaining a reasonable and interpretable fit of the full-information item factor analysis model, the EAP scores are calculated for each examinee. The correlation coefficients between the raw scores and the three EAP score scales are given in Table 3. The raw score for each examinee is simply the sum of the dichotomous responses over the items in a particular scale. On the other hand, the EAP scores were computed on the basis of the multidimensional item response model with estimated slope and location parameters after the Varimax rotation.

Several advantages of the EAP scores over the use of raw scores are evident in Table 3. In examining the correlations, it was found that the EAP score scales have higher correlations with the AOS Scales that have items with high loadings on these scales, as would be expected. It is also clear that the correlation coefficients among the EAP scores were quite small compared to the correlations among the raw scores. The correlations ranged from .455 to .191 for the raw score scales, and from .161 to .094 for the EAP score scales. The orthogonality of the EAP score scales over the multidimensional space indicates that these scores represent distinctive characteristics of the examinees, and provides additional advantages when the EAP scores are used in subsequent analyses. It should also be noted that the EAP scores were calculated for all of the examinees $(N=700)$, while the raw score scales of 50 examinees were excluded because of their missing responses.

In an earlier study, Muraki and Engelhard (1985) conducted several multivariate analyses using four raw score scales and the three EAP score scales. The two independent variables included in this study were the grade level and the gender of the student. In comparing the results of the analyses based on the raw scores and the EAP scores, they found a reasonable amount of agreement between the conclusions based on the separate analyses. The similarity between the analyses is, of course, related to the amount of agreement that was found between the a priori classification of the items into the four AOS Scales and the results of the full-information item factor analysis using the EAP scores. They also noted that the $p$ values were smaller for the analyses using the EAP scores, indicating that the statistical tests appear to be more sensitive with the EAP scores. This is due to the increase in information that is obtained by using all of the data available in the response patterns, which is not used when total scores are analyzed.

Choosing the three-factor model rather than four- or higher dimensional factor models seemed to be appropriate for the present demonstration of actual data analysis as an example. However, as shown in Table 3, the correlation coefficients of the first EAP score with the Honesty and Cooperation scores are

Table 3

Correlations of Raw Score Scales and

the EAP Scores (Lower Triangle) and

Sample Sizes (Upper Triangle)

\begin{tabular}{|c|c|c|c|c|c|c|c|}
\hline & \multirow[b]{2}{*}{ Hо } & \multirow[b]{2}{*}{$\mathrm{CO}$} & \multirow[b]{2}{*}{ CU } & \multirow[b]{2}{*}{ PU } & \multicolumn{3}{|c|}{ EAP Score } \\
\hline & & & & & 1 & 2 & 3 \\
\hline Honesty & 687 & 670 & 667 & 681 & 687 & 687 & 687 \\
\hline Cooperation & .331 & 678 & 660 & 672 & 678 & 678 & 678 \\
\hline Curiosity & .272 & .191 & 672 & 667 & 672 & 672 & 672 \\
\hline Punctuality & .455 & .254 & .324 & 688 & 688 & 688 & 688 \\
\hline 1 & .617 & .751 & .098 & .306 & 700 & 700 & 700 \\
\hline 2 & .302 & .196 & .910 & .353 & .161 & 700 & 700 \\
\hline 3 & .470 & .148 & .152 & .823 & .143 & .094 & 700 \\
\hline
\end{tabular}


not high compared to the correlation either between the second EAP score and the Curiosity Score or between the third EAP score and the Punctuality score. The three-dimensional model of this study may have overlooked other common factors that may be minor but explainable by the content of items of the Honesty and Cooperation scales. Multivariate analyses based on the three-factor solution, therefore, possibly lost information at the price of the reduction of dependent variables. Fitting higher dimensional models to data inevitably makes further analyses and the interpretation of results more complex, but fullinformation factor analysis is useful to explore psychological dimensions which are originally unintended by test item writers.

\section{Conclusions}

The purpose of this study was to present and illustrate the potential applications of EAP scores. Three simulation studies were used to illustrate full-information item factor analysis. The first simulation compared full-information item factor analysis with a conventional tetrachoric factor analysis. This simulation indicated that full-information item factor analysis generally does better in estimating factor loadings in both no-guessing and guessing data sets, especially when item difficulties vary to a great extent. The second simulation study illustrated that the EAP scores based on the Varimax solution were essentially uncorrelated. The third simulation study compared ML and EAP estimates of factor scores. The EAP estimates provided a more reliable reproduction of the simulated abilities than the ML estimates.

Traditional data analysis usually deals with the raw scores obtained from established scales composed of items that are assumed to represent a particular set of constructs. The utilization of EAP scores provides several advantages over raw scores. Some of the specific advantages are

1. The EAP scores are computed on the basis of all of the information available in the examinees' response patterns. Therefore, the EAP scores are more likely to produce a continuous scale than the raw score. This continuity enhances the power of statistical tests used to examine differences between groups.

2. The EAP scores reflect the significant contribution of each item in a test to a specific dimension or factor. Raw score scales reflect only a portion of the items in the test. Therefore, the raw score scales are subject to the potential misclassification of items from established scales. For example, one of the items states "I keep my eyes on my work in the classroom," and it was originally written as an item in the Honesty scale. However, this item has a low factor loading on the first factor and a rather high factor loading on the second factor. Considering this item as equal to the other items in the Honesty scale may distort the conclusions drawn from further analyses of the data.

3. The correlation coefficients among the EAP scores are quite small compared to the correlation among the raw scores. The orthogonality of the EAP scores over the multidimensional space suggests that the EAP scores represent distinctive characteristics of the examinees.

4. Detecting items with poor construct validity becomes easier when full-information factor analysis is used.

5. The raw scores are not generally computable even if one of the responses to the items in a scale is missing. However, the EAP scores can be computed regardless of missing responses.

The interpretation of the multivariate analysis is also simplified with the EAP scores because there are fewer concepts to discuss (Muraki \& Engelhard, 1985). The orthogonality of the EAP scales facilitates the interpretation of the results, since they represent an independent index of each construct. The EAP scores appear to reflect what the test items actually measure, and they are more sensitive to aberrant item behavior. Therefore, the construct validation of psychological or educational measurements based on fullinformation item factor analysis is strongly recommended in the initial item preparation stage. 


\section{References}

Ahrens, J. H., \& Dieter, U. (1972). Computer methods for sampling from the exponential and normal distributions. Communications of the ACM, 15, 873-882.

Bartholomew, D. J. (1980). Factor analysis for categorical data. Journal of the Royal Statistical Society, Series $B, 42,293-321$.

Bock, R. D., \& Aitkin, M. (1981). Marginal maximum likelihood estimation of item parameters: Application of an EM algorithm. Psychometrika, 46, 443-458.

Bock, R. D., Gibbons, R., \& Muraki, E. (1985). Fullinformation item factor analysis. Chicago: National Opinion Research Center.

Bock, R. D., \& Mislevy, R. J. (1982). Adaptive EAP estimation of ability in a microcomputer environment. Applied Psychological Measurement, 6, 431-444.

Bock, R. D., Mislevy, R. J., \& Woodson, C. (1982). The next stage in educational assessment. Educational Researcher, 11(3), 4-11.

Campbell, A., Converse, P. E., \& Rodgers, W. L. (1976). The quality of life. New York: Russel Sage Foundation.

Carroll, J. B. (1945). The effect of difficulty and chance success on correlations between items or between tests. Psychometrika, 10, 1-19.

Christoffersson, A. (1975). Factor analysis of dichotomized variables. Psychometrika, 40, 5-32.

Dempster, A. P., Laird, N. M., \& Rubin, D. B. (1977). Maximum likelihood from incomplete data with the EM algorithm (with discussion). Journal of the Royal Statistical Society, Series B, 34, 1-34.

Divgi, D. R. (1979). Calculation of tetrachoric correlation coefficient. Psychometrika, 40, 5-32.

Engelhard, G. (1985). The discovery of educational goals and outcomes: $A$ view of the latent curriculum of schooling. Unpublished doctoral dissertation, The University of Chicago.

Haberman, J. S. (1977). Log-linear models and frequency tables with small expected cells counts. Annals of Statistics, 5, 1148-1169.

Harman, H. H. (1976). Modern factor analysis. Chicago: The University of Chicago Press.
Kaiser, H. F. (1958). The varimax criterion for analytic rotation in factor analysis. Psychometrika, 23, 187200.

Mislevy, R. J., \& Bock, R. D. (1984). Item operating characteristics of the Armed Services Vocational Aptitude Battery (ASVAB), Form 8A. Chicago: National Opinion Research Center.

Muraki, E., \& Engelhard, G. (1985, March). Affective outcomes of schooling: Full-information item factor analysis of a student questionnaire. Paper presented at the annual meeting of the American Educational Research Association in Chicago.

Muthén, B. (1978). Contributions to factor analysis of dichotomous variables. Psychometrika, 43, 551-560.

Muthén, B. (1984). A general structural equation model with dichotomous, ordered categories, and continuous latent variable indicators. Psychometrika, 49, 115132.

Schrage, L. (1979). A more portable Fortran random generator. ACM Transaction Mathematical Software, 5, 132-138.

Stroud, A. H., \& Sechrest, D. (1966). Gaussian quadrature formulas. Englewood Cliffs NJ: Prentice Hall.

Wilson, D., Wood, R. L., \& Gibbons, R. (1984). TESTFACT: Test scoring and item factor analysis [Computer program]. Mooresville IN: Scientific Software, Inc.

\section{Author's Address}

Send requests for reprints or further information to Eiji Muraki, National Opinion Research Center, 6030 S. Ellis Ave., Chicago IL 60637, U.S.A.

\section{Acknowledgments}

The authors thank R. Darrell Bock for his valuable comments and suggestions. 